\title{
The Fokker-Planck equation
}

\author{
Shambhu N. Sharma † and Hiren G. Patel \\ Department of Electrical Engineering $\dagger$ \\ National Institute of Technology, Surat, India \\ snsvolterra@gmail.com \\ Department of Electrical Engineering $\ddagger$ \\ National Institute of Technology, Surat, India \\ hgp@eed.svnit.ac.in
}

In 1984, H. Risken authored a book (H. Risken, The Fokker-Planck Equation: Methods of Solution, Applications, Springer-Verlag, Berlin, New York) discussing the Fokker-Planck equation for one variable, several variables, methods of solution and its applications, especially dealing with laser statistics. There has been a considerable progress on the topic as well as the topic has received greater clarity. For these reasons, it seems worthwhile again to summarize previous as well as recent developments, spread in literature, on the topic. The Fokker-Planck equation describes the evolution of conditional probability density for given initial states for a Markov process, which satisfies the Itô stochastic differential equation. The structure of the Fokker-Planck equation for the vector case is

$$
\frac{\partial p\left(x, t \mid x_{t_{o}}, t_{0}\right)}{\partial t}=-\operatorname{tr}\left(\frac{\partial f(x, t) p\left(x, t \mid x_{t_{0}}, t_{0}\right)}{\partial x}\right)+\frac{1}{2} \operatorname{tr}\left(\frac{\partial^{2} G G^{T}(x, t) p\left(x, t \mid x_{t_{0}}, t_{0}\right)}{\partial x \partial x^{T}},\right.
$$

where $f\left(x_{t}, t\right)$ is the system non-linearity, $G\left(x_{t}, t\right)$ is termed as the process noise coefficient, and $p\left(x, t \mid x_{t_{0}}, t_{o}\right)$ is the conditional probability density. The Fokker-Planck equation, a prediction density evolution equation, has found its applications in developing prediction algorithms for stochastic problems arising from physics, mathematical control theory, mathematical finance, satellite mechanics, as well as wireless communications. In this chapter, the Authors try to summarize elementary proofs as well as proofs constructed from the standard theories of stochastic processes to arrive at the Fokker-Planck equation. This chapter encompasses an approximate solution method to the Fokker-Planck equation as well as a Fokker-Planck analysis of a Stochastic Duffing-van der Pol (SDvdP) system, which was recently analysed by one of the Authors.

Key words: The Duffing-van der Pol system, the Galerkin approximation, the OrnsteinUhlenbeck process, prediction density, second-order fluctuation equations. 


\section{Introduction}

The stochastic differential equation formalism arises from stochastic problems in diverse field, especially the cases, where stochastic problems are analysed from the dynamical systems' point of view. Stochastic differential equations have found applications in population dynamics, stochastic control, radio-astronomy, stochastic networks, helicopter rotor dynamics, satellite trajectory estimation problems, protein kinematics, neuronal activity, turbulence diffusion, stock pricing, seismology, statistical communication theory, and structural mechanics. A greater detail about stochastic differential equations' applications can be found in Kloeden and Platen (1991). Some of the standard structures of stochastic differential equations are the Itô stochastic differential equation, the Stratonovich stochastic differential equation, the stochastic differential equation involving $p$-differential, stochastic differential equation in Hida sense, non-Markovian stochastic differential equations as well as the Ornstein-Uhlenbeck (OU) process-driven stochastic differential equation. The Itô stochastic differential equation is the standard formalism to analyse stochastic differential systems, since non-Markovian stochastic differential equations can be re-formulated as the Itô stochastic differential equation using the extended phase space formulation, unified coloured noise approximation (Hwalisz et al. 1989). Stochastic differential systems can be analysed using the Fokker-Planck equation (Jazwinski 1970). The Fokker-Planck equation is a parabolic linear homogeneous differential equation of order two in partial differentiation for the transition probability density. The Fokker-Planck operator is an adjoint operator. In literature, the Fokker-Planck equation is also known as the Kolmogorov forward equation. The Kolmogorov forward equation can be proved using mild regularity conditions involving the notion of drift and diffusion coefficients (Feller 2000). The Fokker-Planck equation, definition of the conditional expectation, and integration by part formula allow to derive the evolution of the conditional moment. In the Risken's book, the stochastic differential equation involving the Langevin force was considered and subsequently, the Fokker-Planck equation was derived. The stochastic differential equation with the Langevin force can be regarded as the white noise-driven stochastic differential equation, where the input process satisfies $\left\langle w_{t}\right\rangle=0,\left\langle w_{t} w_{s}\right\rangle=\delta(t-s)$. He considered the approximate solution methods to the scalar and vector Fokker-Planck equations involving change of variables, matrix continued-fraction method, numerical integration method, etc. (Risken 1984, p. 158). Further more, the laser Fokker-Planck equation was derived.

This book chapter is devoted to summarize alternative approaches to derive the FokkerPlanck equation involving elementary proofs as well as proofs derived from the Itô differential rule. In this chapter, the Fokker-Planck analysis hinges on the stochastic differential equation in the Itô sense in contrast to the Langevin sense. From the mathemacians' point of view, the Itô stochastic differential equation involves rigorous interpretation in contrast to the Langevin stochastic differential equation. On the one hand, the stochastic differential equation in Itô sense is described as $d x_{t}=f\left(x_{t}, t\right) d t+G\left(x_{t}, t\right) d B_{t}$, on the other, the Langevin stochastic differential equation assumes the structure $\dot{x}_{t}=f\left(x_{t}, t\right)+G\left(x_{t}, t\right) w_{t}$, where $B_{t}$ and $w_{t}$ are the Brownian and white noises respectively. The white noise can be regarded as an informal 
non-existent time derivative $\dot{B}_{t}$ of the Brownian motion $B_{t}$. Kiyoshi Itô, a famous Japanese mathematician, considered the term ' $d B_{t}{ }^{\prime}=\dot{B}_{t} d t$ and developed Itô differential rule. The results of Itô calculus were published in two seminal papers of Kiyoshi Itô in 1945. The approach of this chapter is different and more exact in contrast to the Risken's book in the sense that involving the Itô stochastic differential equation, introducing relatively greater discussion on the Kolmogorov forward and Backward equations. This chapter discusses a Fokker-Planck analysis of a stochastic Duffing-van der Pol system, an appealing case, from the dynamical systems' point of view as well.

This chapter is organised as follows: (i) section 2 discusses the evolution equation of the prediction density for the Itô stochastic differential equation. A brief discussion about approximate methods to the Fokker-Planck equation, stochastic differential equation is also given in section 2 (ii) in section 3, the stochastic Duffing-van der Pol system was analysed to demonstrate a usefulness of the Fokker-Planck equation. (iii) Section 4 is about the numerical simulation of the mean and variance evolutions of the SDvdP system. Concluding remarks are given in section (5).

\section{Evolution of conditional probability density}

The Fokker-Planck equation describes the evolution of conditional probability density for given initial states for the Itô stochastic differential system. The equation is also known as the prediction density evolution equation, since it can be utilized to develop prediction algorithms, especially where observations are not available at every time instant. One of the potential applications of the Fokker-Planck equation is to develop estimation algorithms for the satellite trajectory estimation. This chapter summarizes four different proofs to arrive at the Fokker-Planck equation. The first two proofs can be regarded as elementary proofs and the last two utilize the Itô differential rule. Moreover, the Fokker-Planck equation for the OU process-driven stochastic differential equation is discussed here, where the input process has non-zero, finite, relatively smaller correlation time.

The first proof of this chapter begins with the Chapman-Kolmogorov equation. The Chapman-Kolmogorov equation is a consequence of the theory of the Markov process. This plays a key role in proving the Kolmogorov backward equation (Feller 2000). Here, we describe briefly the Chapman-Kolmogorov equation and subsequently, the concept of the conditional probability density as well as transition probability density are introduced to derive the evolution of conditional probability density for the non-Markov process. The Fokker-Planck equation becomes a special case of the resulting equation. The conditional probability density

$$
p\left(x_{1}, x_{2} \mid x_{3}\right)=p\left(x_{1} \mid x_{2}, x_{3}\right) \ldots p\left(x_{2} \mid x_{3}\right) .
$$

Consider the random variables $x_{t_{1}}, x_{t_{2}}, x_{t_{3}}$ at the time instants $t_{1}, t_{2}, t_{3}$, where $t_{1}>t_{2}>t_{3}$ and take values $x_{1}, x_{2}, x_{3}$. In the theory of the Markov process, the above can be re-stated as

$$
p\left(x_{1}, x_{2} \mid x_{3}\right)=p\left(x_{1} \mid x_{2}\right) p\left(x_{2} \mid x_{3}\right),
$$


integrating over the variable $x_{2}$, we have

$$
p\left(x_{1} \mid x_{3}\right)=\int p\left(x_{1} \mid x_{2}\right) p\left(x_{2} \mid x_{3}\right) d x_{2}
$$

introducing the notion of the transition probability density and time instants

$$
q_{t+s}\left(x_{1}, x_{3}\right)=\int q_{t}\left(x_{1}, x_{2}\right) q_{s}\left(x_{2}, x_{3}\right) d x_{2}
$$

Consider the multi-dimensional probability density $p\left(x_{1}, x_{2}\right)=p\left(x_{1} \mid x_{2}\right) p\left(x_{2}\right)$ and integrating over the variable $x_{2}$, we have

$$
p\left(x_{1}\right)=\int p\left(x_{1} \mid x_{2}\right) p\left(x_{2}\right) d x_{2}
$$

or

$$
p\left(x_{1}\right)=\int q_{t_{1}, t_{2}}\left(x_{1}, x_{2}\right) p\left(x_{2}\right) d x_{2},
$$

where $q_{t_{1}, t_{2}}\left(x_{1}, x_{2}\right)$ is the transition probability density and $t_{1}>t_{2}$. The transition probability density $q_{t_{1}, t_{2}}\left(x_{1}, x_{2}\right)$ is the inverse Fourier transform of the characteristic function $E e^{i u\left(x_{t_{1}}-x_{t_{2}}\right)}$, i.e.

$$
q_{t_{1}, t_{2}}\left(x_{1}, x_{2}\right)=\frac{1}{2 \pi} \int e^{-i u\left(x_{1}-x_{2}\right)} E e^{i u\left(x_{t_{1}}-x_{t_{2}}\right)} d u .
$$

Equation (1) in combination with equation (2) leads to

$$
p\left(x_{1}\right)=\frac{1}{2 \pi} \int e^{-i u\left(x_{1}-x_{2}\right)}\left(E e^{i u\left(x_{t_{1}}-x_{t_{2}}\right)}\right) p\left(x_{2}\right) d x_{2} d u \text {. }
$$

The characteristic function is the moment generating function, the characteristic function $E e^{i u\left(x_{t_{1}}-x_{t_{1}}\right)}=\sum_{0 \leq n} \frac{(i u)^{n}}{n !}\left\langle\left(x_{t_{1}}-x_{t_{2}}\right)^{n}\right\rangle$. After introducing the definition of the characteristic function, equation (3) can be recast as

$$
\begin{gathered}
p\left(x_{1}\right)=\frac{1}{2 \pi} \int e^{-i u\left(x_{1}-x_{2}\right)}\left(\sum_{0 \leq n} \frac{(i u)^{n}}{n !}\left\langle\left(x_{t_{1}}-x_{t_{2}}\right)^{n}\right\rangle\right) p\left(x_{2}\right) d x_{2} d u \\
=\sum_{0 \leq n} \int \frac{1}{n !}\left(\frac{1}{2 \pi} \int(i u)^{n} e^{-i u\left(x_{1}-x_{2}\right)} d u\right)\left\langle\left(x_{t_{1}}-x_{t_{2}}\right)^{n}\right\rangle p\left(x_{2}\right) d x_{2} .
\end{gathered}
$$


The term $\frac{1}{2 \pi} \int e^{-i u\left(x_{1}-x_{2}\right)}(i u)^{n} d u=\left(-\frac{\partial}{\partial x_{1}}\right)^{n} \delta\left(x_{1}-x_{2}\right)$ and leads to the probability density

$$
p\left(x_{1}\right)=\sum_{0 \leq n} \int \frac{1}{n !}\left(-\frac{\partial}{\partial x_{1}}\right)^{n} \delta\left(x_{1}-x_{2}\right)\left\langle\left(x_{t_{1}}-x_{t_{2}}\right)^{n}\right\rangle p\left(x_{2}\right) d x_{2} .
$$

For the short hand notation, introducing the notion of the stochastic process, taking $x_{1}=x_{\tau}, x_{2}=x$, where the time instants $t_{1}=t+\tau, t_{2}=t$, equation (4) can be recast as

$$
\begin{aligned}
p\left(x_{\tau}\right) & =\sum_{0 \leq n} \int \frac{1}{n !}\left(-\frac{\partial}{\partial x_{\tau}}\right)^{n} \delta\left(x_{\tau}-x\right)\left\langle\left(x_{\tau}-x\right)^{n}\right\rangle p(x) d x \\
& =\sum_{0 \leq n} \int \frac{1}{n !}\left(-\frac{\partial}{\partial x_{\tau}}\right)^{n} \delta\left(x_{\tau}-x\right) k_{n}(x) \tau p(x) d x
\end{aligned}
$$

where $\left\langle\frac{\left(x_{\tau}-x\right)^{n}}{\tau}\right\rangle=k_{n}(x)$ and the time interval condition $\tau \rightarrow 0$ leads to

$$
\operatorname{Lt}_{\tau \rightarrow 0} \frac{p\left(x_{\tau}\right)-p(x)}{\tau}=\sum_{1 \leq n} \frac{1}{n !}\left(-\frac{\partial}{\partial x_{\tau}}\right)^{n} k_{n}(x) p(x),
$$

or

$$
\dot{p}(x)=\sum_{1 \leq n} \frac{1}{n !}\left(-\frac{\partial}{\partial x}\right)^{n} k_{n}(x) p(x)
$$

Note that the above density evolution equation is derived for the arbitrary stochastic process $X=\left(x_{t}, 0 \leq t<\infty\right)$. Here, the arbitrary process means that there is no restriction imposed on the process while deriving the density evolution equation and can be regarded as the non-Markov process. Consider a Markov process, which satisfies the Itô stochastic differential equation, the evolution of conditional probability density retains only the first two terms $k_{1}(x)$ and $k_{2}(x)$, which is a direct consequence of the stochastic differential rule for the Itô stochastic differential equation in combination with the definition $\left\langle\frac{\left(x_{\tau}-x\right)^{n}}{\tau}\right\rangle=k_{n}(x)$. As a result of these, the evolution of conditional probability density for the scalar stochastic differential equation of the form

$$
d x_{t}=f\left(x_{t}, t\right) d t+g\left(x_{t}, t\right) d B_{t},
$$


leads to the Fokker-Planck equation,

$$
\dot{p}(x)=-\frac{\partial}{\partial x} f(x, t) p(x)+\frac{1}{2} \frac{\partial^{2} g^{2}(x, t)}{\partial x^{2}} p(x),
$$

where $k_{1}(x)=f(x, t), k_{2}(x)=g^{2}(x, t)$. The Fokker-Planck equation can be recast as $d p(x)=L p(x) d t$, where the vector version of the Fokker-Planck operator

$$
L(.)=-\sum_{i} \frac{\partial}{\partial x_{i}} f_{i}(x, t)(.)+\frac{1}{2} \sum_{i, j} \frac{\partial^{2}\left(G G^{T}\right)_{i j}(x, t)(.)}{\partial x_{i} \partial x_{j}} .
$$

The Fokker-Planck operator is an adjoint operator, since $\langle\phi, L(p)\rangle=\left\langle L^{\prime} \phi, p\right\rangle$, where $L^{\prime}($.$) is the Kolmogorov backward operator. This property is utilized in deriving the$ evolution $d \hat{\phi}\left(x_{t}\right)$ of the conditional moment (Jazwinski 1970). The Fokker-Planck equation is also known as the Kolmogorov Forward equation.

The second proof of this chapter begins with the Green function, the Kolmogorov forward and backward equations involve the notion of the drift and diffusion coefficients as well as mild regularity conditions (Feller 2000). The drift and diffusion coefficients are regarded as the system non-linearity and the 'stochastic perturbation in the variance evolution' respectively in noisy dynamical system theory. Here, we explain briefly about the formalism associated with the proof of the Kolmogorov forward and backward equations. Consider the Green's function

$$
u_{t}(x)=\int q_{t}(x, y) u_{0}(y) d y
$$

where $q_{t}(x, y)$ is the transition probability density, $u_{t}(x)$ is a scalar function, $x$ is the initial point and $y$ is the final point. Equation (5) is modified at the time duration $t+h$ as

$$
u_{t+h}(x)=\int q_{t+h}(x, y) u_{0}(y) d y .
$$

The Chapman-Kolmogorov equation can be stated as

$$
q_{t+h}(x, y)=\int q_{t}(x, \varsigma) q_{h}(\varsigma, y) d \varsigma
$$

Making the use of equations (6)-(7) and the Taylor series expansion with mild regularity conditions leads to 


$$
\frac{\partial u_{t}(x)}{\partial t}=b(x) \frac{\partial u_{t}(x)}{\partial x}+\frac{1}{2} a(x) \frac{\partial^{2} u_{t}(x)}{\partial x^{2}}
$$

where $\frac{\partial u_{t}(x)}{\partial t}=L_{h \rightarrow 0} \frac{u_{t+h}-u_{t}}{h}, b(x)$ and $a(x)$ are the drift and diffusion coefficients respectively (Feller 2000), and the detailed proof of equation (8) can be found in a celebrated book authored by Feller (2000). For the vector case, the Kolmogorov backward equation can be recast as

$$
\frac{\partial u_{t}(x)}{\partial t}=\sum b_{i}(x) \frac{\partial u_{t}(x)}{\partial x_{i}}+\sum \frac{1}{2} a_{i j}(x) \frac{\partial^{2} u_{t}(x)}{\partial x_{i} \partial x_{j}}
$$

where the summation is extended for $1 \leq i \leq n, 1 \leq j \leq n$. From the dynamical systems' point of view, the vector case of the Kolmogorov backward equation can be reformulated as

$$
\frac{\partial u_{t}(x)}{\partial t}=\sum f_{i}(x, t) \frac{\partial u_{t}(x)}{\partial x_{i}}+\frac{1}{2} \sum(G G)_{i j}(x, t) \frac{\partial^{2} u_{t}(x)}{\partial x_{i} \partial x_{j}}
$$

where the mappings $f$ and $G$ are the system non-linearity and process noise coefficient matrix respectively and the Kolmogorov backward operator

$$
L^{\prime}(.)=\sum f_{i}(x, t) \frac{\partial(.)}{\partial x_{i}}+\sum_{i} \frac{1}{2}(G G)_{i j}(x, t) \frac{\partial^{2}(.)}{\partial x_{i} \partial x_{j}} \text {. }
$$

Note that the Kolmogorov backward equation is a parabolic linear homogeneous differential equation of order two in partial differentiation, since the backward operator is a linear operator and the homogeneity condition holds. The Kolmogorov forward equation can be derived using the relation

$$
v_{s}(y)=\int q_{s}(x, y) v_{0}(x) d x
$$

in combination with integration by part formula as well as mild regularity conditions (Feller 2000) lead to the expression

$$
\frac{\partial v_{s}(y)}{\partial s}=-\frac{\partial b(y) v_{s}(y)}{\partial y}+\frac{1}{2} \frac{\partial^{2} a(y) v_{s}(y)}{\partial y^{2}}
$$

The terms $b(y)$ and $a(y)$ of equation (9) have similar interpretations as the terms of equation (8). The vector version of equation (9) is 


$$
\frac{\partial v_{s}(y)}{\partial s}=-\sum_{i} \frac{\partial b_{i}(y) v_{s}(y)}{\partial y_{i}}+\frac{1}{2} \sum_{i, j} \frac{\partial^{2} a_{i j}(y) v_{s}(y)}{\partial y_{i} \partial y_{j}}
$$

and the Kolmogorov forward operator $L()=.-\sum_{i} \frac{\partial b_{i}(y)(.)}{\partial y_{i}}+\frac{1}{2} \sum_{i, j} \frac{\partial^{2} a_{i j}(y)(.)}{\partial y_{i} \partial y_{j}}$. For $b_{i}=f_{i}$ and $a_{i j}=\left(G G^{T}\right)_{i j}$, the Kolmogorov forward operator assumes the structure of the Fokker-Planck operator and is termed as the Kolmogorov-Fokker-Planck operator.

The third proof of the chapter explains how the Fokker-Planck equation can be derived using the definition of conditional expectation and Itô differential rule.

$$
E \phi\left(x_{t+d t}\right)=E\left(E\left(\phi\left(x_{t+d t}\right) \mid x_{t}=x\right)\right) .
$$

The Taylor series expansion of the scalar function $\phi\left(x_{t+d t}\right)=\sum_{m} \frac{\left(d x_{t}\right)^{m}}{m !} \phi^{m}\left(x_{t}\right)$ and

$$
E\left(\phi\left(x_{t+d t}\right) \mid x_{t}=x\right)=\sum_{0 \leq m \leq 2} \frac{\mu_{m}(x, t)}{m !} \phi^{m}(x)
$$

where $\mu_{m}(x, t)=E\left(\left(d x_{t}\right)^{m} \mid x_{t}=x\right)$ and the summing variable $m$ takes values upto two for the Brownian motion process-driven stochastic differential equation that can be explained via the Itô differential rule. Equation (10) in conjunction with equation (11) leads to

$$
E \phi\left(x_{t+d t}\right)=\sum_{0 \leq m \leq 2} E\left(\frac{\mu_{m}(x, t)}{m !} \phi^{m}(x)\right)
$$

the definition of 'expectation' leads to the following expression:

$$
\int \phi(x) p(x, t+d t) d x=\sum_{0 \leq m \leq 2} \int \frac{\mu_{m}(x, t)}{m !} \phi^{m}(x) p(x, t) d x
$$

the integration by part, applying to equation (12), leads to the Fokker-Planck equation,

$$
\frac{\partial p(x, t)}{\partial t}=\sum_{1 \leq m \leq 2} \frac{(-1)^{m}}{m !} \frac{\partial^{m} \mu_{m}(x, t) p(x, t)}{\partial x^{m}}
$$

where

$$
\mu_{1}=f(x, t), \mu_{2}=g^{2}(x, t) .
$$


Finally, we derive the Fokker-Planck equation using the concept of the evolution of the conditional moment and the conditional characteristic function. Consider the state vector $x_{t} \in U, \phi: U \rightarrow R$, i.e. $\phi\left(x_{t}\right) \in R$, and the phase space $U \subset R^{n}$. The state vector $x_{t}$ satisfies the Itô SDE as well. Suppose the function $\phi\left(x_{t}\right)$ is twice differentiable. The evolution $d \hat{\phi}\left(x_{t}\right)$ of the conditional moment is the standard formalism to analyse stochastic differential systems. Further more, $d \hat{\phi}\left(x_{t}\right)=E\left(d \phi\left(x_{t}\right) \mid x_{t_{0}}, t_{0}\right)$ holds. A greater detail can be found in Sharma (2008). The stochastic evolution $d \phi\left(x_{t}\right)$ of the scalar function $\phi\left(x_{t}\right)$ (Sage and Melsa 1971) can be stated as

$$
\begin{gathered}
d \phi\left(x_{t}\right)=\left(\sum_{i} \frac{\partial \phi\left(x_{t}\right)}{\partial x_{i}} f_{i}\left(x_{t}, t\right)+\frac{1}{2} \sum_{i}\left(G G^{T}\right)_{i i}\left(x_{t}, t\right) \frac{\partial^{2} \phi\left(x_{t}\right)}{\partial x_{i}^{2}}\right. \\
\left.+\sum_{i<j}\left(G G^{T}\right)_{i j}\left(x_{t}, t\right) \frac{\partial^{2} \phi\left(x_{t}\right)}{\partial x_{i} \partial x_{j}}\right) d t+\sum_{1 \leq i \leq n, 1 \leq \gamma \leq r} \frac{\partial \phi\left(x_{t}\right)}{\partial x_{i}} G_{i \gamma}\left(x_{t}, t\right) d B_{\gamma},
\end{gathered}
$$

thus

$$
d \widehat{\phi}\left(x_{t}\right)=(\overbrace{\sum_{p} f_{p}\left(x_{t}, t\right) \frac{\partial \phi\left(x_{t}\right)}{\partial x_{p}}}^{\Lambda}+\frac{1}{2} \sum_{p}(\overbrace{\left(G G^{T}\right)_{p p}\left(x_{t}, t\right) \frac{\partial^{2} \phi\left(x_{t}\right)}{\partial x_{p}^{2}}}^{\Lambda}+\overbrace{\left.\sum_{p<q}\left(G G^{T}\right)_{p q}\left(x_{t}, t\right) \frac{\partial^{2} \phi\left(x_{t}\right)}{\partial x_{p} \partial x_{q}}\right) d t .}^{\Lambda}
$$

Note that the expected value of the last term of the right-hand side of equation (13) vanishes, i.e. $\left\langle G_{i \gamma}\left(x_{t}, t\right) d B_{\gamma}\right\rangle=0$. Consider $\phi\left(x_{t}\right)=e^{S^{T} x_{t}}$, the evolution of the characteristic function becomes

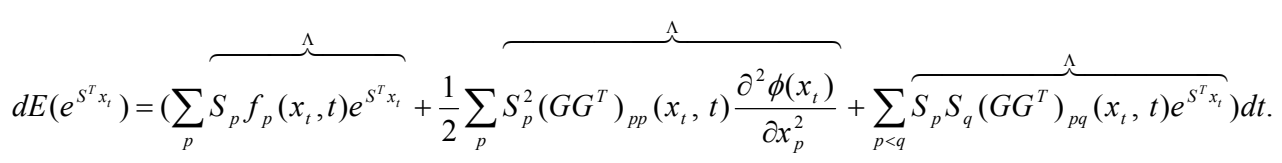

Making the use of the definition of the characteristic function as well as the integration by part formula, we arrive at the Fokker-Planck equation.

The Kushner equation, the filtering density evolution equation for the Itô stochastic differential equation, is a 'generalization' of the Fokker-Planck equation. The Kushner equation is a partial-integro stochastic differential equation, i.e.

$$
d p=L(p) d t+(h-\widehat{h})^{T} \varphi_{n}^{-1}\left(d z_{t}-\hat{h} d t\right) p
$$


where $L($.$) is the Fokker-Planck operator, p=p\left(x, t \mid z_{\tau}, t_{0} \leq \tau \leq t\right)$, the observation $z_{t}=\int_{t_{0}}^{t} h\left(x_{\tau}, \tau\right) d \tau+B_{t}$, and $h\left(x_{t}, t\right)$ is the measurement non-linearity. Harald $\mathrm{J}$ Kushner first derived the expression of the filtering density and subsequently, the filtering density evolution equation using the stochastic differential rule (Jazwinski 1970). LiptserShiryayev discovered an alternative proof of the filtering density evolution, equation (14), involving the following steps: (i) derive the stochastic evolution $d \overbrace{\phi\left(x_{t}\right)}^{\Lambda}$ of the conditional moment, where $\overbrace{\phi\left(x_{t}\right)}^{\Lambda}=E\left(\phi\left(x_{t}\right) \mid z_{\tau}, t_{0} \leq \tau \leq t\right)$ (ii) subsequently, the stochastic evolution of the conditional characteristic function can be regarded as a special case of the conditional moment evolution, where $\phi\left(x_{t}\right)=e^{S^{T} x_{t}}$ (iii) the definition of the conditional expectation as well as integration by part formula lead to the filtering density evolution equation, see Liptser and Shiryayev (1977). RL Stratonovich developed the filtering density evolution for stochastic differential equation involving the $\frac{1}{2}$-differential as well. For this reason, the filtering density evolution equation is also termed as the Kushner-Stratonovich equation.

Consider the stochastic differential equation of the form

$$
\dot{x}_{t}=f\left(x_{t}\right)+g\left(x_{t}\right) \xi_{t},
$$

where $\xi_{t}$ is the Ornstein-Uhlenbeck process and generates the process $x_{t}$, a non-Markov process. The evolution of conditional probability density for the non-Markov process with the input process with a non-zero, finite, smaller correlation time $\tau_{c o r}$, i.e. $0<\tau_{\text {cor }}<<1$, reduces to the Fokker-Planck equation. One of the approaches to arrive at the Fokker-Planck equation for the OU process-driven stochastic differential equation with smaller correlation time is function calculus. The function calculus approach involves the notion of the functional derivative. The evolution of conditional probability density for the output process $x_{t}$, where the input process $\xi_{t}$ is a zero mean, stationary and Gaussian process, can be written (Hänggi 1995, p.85) as

$$
\dot{p}_{t}(x)=-\frac{\partial f(x) p}{\partial x}+\frac{\partial}{\partial x} g(x)\left(\frac{\partial}{\partial x} \int_{t_{o}}^{t} C_{2}(t-s)\left\langle\delta\left(x_{t}-x\right) \frac{\delta x_{t}}{\delta \xi_{s}}\right\rangle d s\right)
$$


where the second-order cumulant of the zero mean, stationary and Gaussian process is $C_{2}(t, s)=\operatorname{cov}\left(\xi_{t}, \xi_{s}\right)=R_{\xi \xi}(t-s)$ and $\frac{\delta x_{t}}{\delta \xi_{s}}$ is the functional derivative of the process $x_{t}$ with respect to the input process $\xi_{s}$. The integral counterpart of equation (15) is

$$
x_{t}=x_{t_{0}}+\int_{t_{0}}^{t} f\left(x_{\tau}\right)+g\left(x_{\tau}\right) \xi_{\tau} d \tau
$$

The functional derivative $\frac{\delta x_{t}}{\delta \xi_{s}}$ depends on the time interval $s \leq \tau \leq t$ and can be stated as

$$
\begin{aligned}
\frac{\delta x_{t}}{\delta \xi_{s}} & =\int_{s}^{t}\left(f^{\prime}\left(x_{\tau}\right) \frac{\delta x_{\tau}}{\delta \xi_{s}}+g^{\prime}\left(x_{\tau}\right) \frac{\delta x_{t}}{\delta \xi_{s}} \xi_{\tau}+g\left(x_{\tau}\right) \frac{\delta \xi_{\tau}}{\delta \xi_{s}}\right) d \tau \\
& =g\left(x_{s}\right)+\int_{s}^{t}\left(f^{\prime}\left(x_{\tau}\right) \frac{\delta x_{\tau}}{\delta \xi_{s}}+g^{\prime}\left(x_{\tau}\right) \frac{\delta x_{\tau}}{\delta \xi_{s}} \xi_{\tau}\right) d \tau
\end{aligned}
$$

Making the repetitive use of the expression $\frac{\delta x_{t}}{\delta \xi_{s}}$ within the integral sign of equation (17), we have

$$
\begin{gathered}
\frac{\delta x_{t}}{\delta \xi_{s}}=g\left(x_{s}\right) \exp \left(\int_{s}^{t} \frac{\partial \dot{x}_{\tau}}{\partial x_{\tau}} d \tau\right) \\
=g\left(x_{s}\right) \exp \left(\int_{s}^{t}\left(f^{\prime}\left(x_{\tau}\right)+g^{\prime}\left(x_{\tau}\right) \xi_{\tau} d \tau\right)\right) .
\end{gathered}
$$

More over, the time derivative of the process noise coefficient $g\left(x_{t}\right)$ of equation (15) can be written as

$$
\begin{gathered}
\dot{g}\left(x_{t}\right)=g^{\prime}\left(x_{t}\right) \dot{x}_{t} \\
=g^{\prime}\left(x_{t}\right)\left(f\left(x_{t}\right)+g\left(x_{t}\right) \xi_{t}\right),
\end{gathered}
$$

after some calculations, the integral counterpart of the above equation can be stated as

$$
g\left(x_{s}\right)=g\left(x_{t}\right) \exp \left(-\left(\int_{s}^{t}\left(\frac{g^{\prime}\left(x_{\tau}\right) f\left(x_{\tau}\right)}{g\left(x_{\tau}\right)}+g^{\prime}\left(x_{\tau}\right) \xi_{\tau}\right) d \tau\right)\right)
$$


Equation (18) in combination with equation (19) leads to

$$
\frac{\delta x_{t}}{\delta \xi_{s}}=g\left(x_{t}\right) \exp \left(\int_{s}^{t}\left(f^{\prime}\left(x_{\tau}\right)-g^{\prime}\left(x_{\tau}\right) \frac{f\left(x_{\tau}\right)}{g\left(x_{\tau}\right)}\right) d \tau\right)
$$

Furthermore, the Taylor series expansion of the functional derivative $\frac{\delta x_{t}}{\delta \xi_{s}}$ in powers of $(s-t)$ can be stated as

$$
\frac{\delta x_{t}}{\delta \xi_{s}}=\frac{\delta x_{t}}{\delta \xi_{t}}+(s-t)\left(\frac{\partial}{\partial s}\left(\frac{\delta x_{t}}{\delta \xi_{s}}\right) \mid s=t\right)+O\left((s-t)^{2}\right) .
$$

From equation (20), we have

$$
\begin{gathered}
\frac{\delta x_{t}}{\delta \xi_{t}}=g\left(x_{t}\right) \\
\frac{\partial}{\partial s} \frac{\delta x_{t}}{\delta \xi_{s}} \mid s=t=-g\left(x_{t}\right)\left(f^{\prime}\left(x_{t}\right)-g^{\prime}\left(x_{t}\right) \frac{f\left(x_{t}\right)}{g\left(x_{t}\right)}\right) .
\end{gathered}
$$

After retaining the first two terms of the right-hand side of equation (21) and equations (22)(23) in combination with equation (21) lead to

$$
\begin{aligned}
& \frac{\delta x_{t}}{\delta \xi_{s}}=g\left(x_{t}\right)+(t-s) g\left(x_{t}\right)\left(f^{\prime}\left(x_{t}\right)-g^{\prime}\left(x_{t}\right) \frac{f\left(x_{t}\right)}{g\left(x_{t}\right)}\right) \\
& =g\left(x_{t}\right)\left(1+(t-s)\left(\frac{f^{\prime}\left(x_{t}\right) g\left(x_{t}\right)-g^{\prime}\left(x_{t}\right) f\left(x_{t}\right)}{g\left(x_{t}\right)}\right)\right)
\end{aligned}
$$

thus

$$
\left\langle\delta\left(x_{t}-x\right) \frac{\delta x_{t}}{\delta \xi_{s}}\right\rangle=g(x)\left(1+(t-s) g(x)\left(\frac{f(x)}{g(x)}\right)^{\prime}\right) p(x)
$$

The autocorrelation $R_{\xi \xi}(t-s)$ of the OU process satisfying the stochastic differential equation $d \xi_{t}=\frac{-1}{\tau_{c o r}} \xi_{t} d t+\frac{\sqrt{2 D}}{\tau_{c o r}} d B_{t}$ becomes

$$
R_{\xi \xi}(t-s)=\frac{D}{\tau_{c o r}} e^{-\frac{|t-s|}{\tau_{c o r}}}
$$


Equations (24)-(25) in conjunction with equation (16) give

$$
\dot{p}(x)=-\frac{\partial}{\partial x} f p+D \frac{\partial}{\partial x}\left(g \frac{\partial}{\partial x} g\left(1+\tau_{c o r} g\left(\frac{f}{g}\right)^{\prime}\right) p\right) .
$$

The Kolmogorov-Fokker-Planck equation and the Kolmogorov backward equation are exploited to analyse the Ito stochastic differential equation by deriving the evolution of the conditional moment. The evolutions of conditional mean and variance are the special cases of the conditional moment evolution. The conditional mean and variance evolutions are infinite dimensional as well as involve higher-order moments. For these reasons, approximate mean and variance evolutions are derived and examined involving numerical experiments. Alternatively, the Carleman linearization to the exact stochastic differential equation resulting the bilinear stochastic differential equation has found applications in developing the approximate estimation procedure. The Carleman linearization transforms a finite dimensional non-linear system into a system of infinite dimensional linear systems (Kowalski and Steeb 1991).

The exact solution of the Fokker-Planck equation is possible for the simpler form of the stochastic differential equation, e.g.

$$
d x_{t}=a d B_{t}
$$

The Fokker-Planck equation for equation (26) becomes

$$
\frac{\partial p\left(x, t \mid x_{t_{0}}, t_{0}\right)}{\partial t}=\frac{1}{2} a^{2} \frac{\partial^{2} p\left(x, t \mid x_{t_{0}}, t_{0}\right)}{\partial x^{2}} .
$$

Consider the process $N\left(0, a^{2} t\right)$ and its probability density

$$
p\left(x, t \mid x_{t_{0}}, t_{0}\right)=\frac{1}{\sqrt{2 \pi a^{2} t}} e^{-\frac{x^{2}}{2 a^{2} t}}
$$

satisfies equation (26). However, the closed-form solution to the Fokker-Planck equation for the non-linear stochastic differential equation is not possible, the approximate solution to the Fokker-Planck equation is derived. The Galerkin approximation to the Fokker-Planck equation received some attention in literature. The Galerkin approximation can be applied to the Kushner equation as well. More generally, the usefulness of the Galerkin approximation to the partial differential equation and the stochastic differential equation for the approximate solution can be explored. The theory of the Galerkin approximation is grounded on the orthogonal projection lemma. For a greater detail, an authoritative book, computational Galerkin methods, authored by C A J Fletcher can be consulted (Fletcher 1984). 


\section{A stochastic Duffing-van der Pol system}

The second-order fluctuation equation describes a dynamical system in noisy environment. The second-order fluctuation equation can be regarded as

$$
\ddot{x}_{t}=F\left(t, x_{t}, \dot{x}_{t}, \dot{B}_{t}\right) \text {. }
$$

The phase space formulation allows transforming a single equation of order $n$ into a system of $n$ first-order differential equations. Choose $x_{t}=x_{1}$

$$
\begin{gathered}
\dot{x}_{1}=x_{2}, \\
\dot{x}_{2}=F\left(t, x_{1}, x_{2}, \dot{B}_{t}\right),
\end{gathered}
$$

by considering a special case of the above system of equations, we have

$$
\begin{gathered}
d x_{1}=x_{2} d t, \\
d x_{2}=f_{2}\left(t, x_{1}, x_{2}\right) d t+g_{2}\left(t, x_{1}, x_{2}\right) d B_{t},
\end{gathered}
$$

in matrix-vector format

$$
d \xi_{t}=f\left(t, x_{1}, x_{2}\right) d t+G\left(t, x_{1}, x_{2}\right) d B_{t}
$$

where

$$
\xi_{t}=\left(\xi_{1}, \xi_{2}\right)^{T}=\left(x_{1}, x_{2}\right)^{T}, f\left(t, \xi_{t}\right)=\left(x_{2}, f_{2}\right)^{T}, G\left(t, \xi_{t}\right)=\left(0_{2}, g_{2}\right)^{T}
$$

The stochastic Duffing-van der Pol system can be formulated in the form of equation (27) (Sharma 2008), where

$$
f\left(\xi_{t}, t\right)=\left(\begin{array}{l}
x_{2} \\
\left.\alpha x_{1}+\beta x_{2}-a x_{1}^{3}-b x_{2} x_{1}^{2}\right)
\end{array}\right), G\left(\xi_{t}, t\right)=\left(\begin{array}{l}
0 \\
\sigma_{B} x_{1}^{n}
\end{array}\right)
$$

and $G\left(\xi_{t}, t\right)$ is the process noise coefficient matrix. The Fokker-Planck equation can be stated as (Sage and Melsa1971, p.100)

$$
\frac{\partial p\left(\xi, t \mid \xi_{t_{0}}, t_{0}\right)}{\partial t}=-\sum_{i} \frac{\partial f_{i}(\xi, t) p\left(\xi, t \mid \xi_{t_{0}}, t_{0}\right)}{\partial \xi_{i}}
$$




$$
+\frac{1}{2} \sum_{i, j} \frac{\partial^{2}\left(G G^{T}\right)_{i, j}(\xi, t) p\left(\xi, t \mid \xi_{t_{0}}, t_{0}\right)}{\partial \xi_{i} \partial \xi_{j}}
$$

where $\xi=\left(x_{1}, x_{2}, \ldots, x_{n}\right)^{T}$. Equation (29) in combination with equation (28) leads to the Fokker-Planck equation for the stochastic system of this chapter, i.e.

$$
\begin{gathered}
\frac{\partial p}{\partial t}=-x_{2} \frac{\partial p}{\partial x_{1}}-\alpha x_{1} \frac{\partial p}{\partial x_{2}}-\beta x_{2} \frac{\partial p}{\partial x_{2}}-\beta p+a x_{1}^{3} \frac{\partial p}{\partial x_{2}}+b x_{1}^{2} p \\
+b x_{2} x_{1}^{2} \frac{\partial p}{\partial x_{2}}+\frac{\sigma_{B}^{2}}{2}(2 n)(2 n-1) x_{1}^{2 n-2} p .
\end{gathered}
$$

Alternatively, the stochastic differential system can be analysed qualitatively involving the Itô differential rule, see equation (13) of the chapter. The energy function for the stochastic system of this chapter is

$$
E\left(x_{1}, x_{2}\right)=\frac{1}{2} x_{2}^{2}-\alpha \frac{x_{1}^{2}}{2}+a \frac{x_{1}^{4}}{4} .
$$

From equations (13), (28), and (30), we obtain

$$
\begin{aligned}
d E\left(x_{1}, x_{2}\right)=\left(\left(-\alpha x_{1}+a x_{1}^{3}\right) x_{2}+\right. & \left.x_{2}\left(\alpha x_{1}+\beta x_{2}-a x_{1}^{3}-b x_{2} x_{1}^{2}\right)+\frac{1}{2} \sigma_{B}^{2} x_{1}^{2 n}\right) d t \\
& +\sigma_{B} x_{1}^{n} d w_{t} .
\end{aligned}
$$

After a simple calculation, we have the following SDE:

$$
d E=\left(\beta x_{2}^{2}-b x_{2}^{2} x_{1}^{2}+\frac{1}{2} \sigma_{B}^{2} x_{1}^{2 n}+\frac{1}{2} \sigma_{u}^{2}\right) d t+\sigma_{B} x_{1}^{n} d w_{t}+\sigma_{u} d v_{t} .
$$

The qualitative analysis of the stochastic problem of this chapter using the multidimensional Itô differential rule illustrates the contribution of diffusion parameters to the stochastic evolution of the energy function. The energy evolution equation suggests the system will exhibit either increasing oscillations or decreasing depending on the choice of the parameters $\beta, b$, and the diffusion parameters $\sigma_{B}, \sigma_{u}$. The numerical experiment also confirms the qualitative analysis of this chapter, see figures (1)-(2). This chapter discusses a Fokker-Planck analysis of the SDvdP system, recently analysed and published by one of the Authors (Sharma 2008).

Making use of the Fokker-Planck equation, Kolmogorov backward equation, the evolutions of condition mean and variances (Jazwinski 1970, p. 363) can be stated as 


$$
\begin{gathered}
d \widehat{x}_{i}=\overbrace{f_{i}\left(x_{t}, t\right)}^{\Lambda} d t, \\
d P_{i j}=(\overbrace{\left(x_{i} f_{j}\right.}^{\Lambda}-\hat{x}_{i} \hat{f}_{j}+\overbrace{f_{i} x_{j}}^{\Lambda}-\hat{f}_{i} \hat{x}_{j}+\overbrace{\left(G G^{T}\right)_{i j}\left(x_{t}, t\right)}^{\Lambda} d t,
\end{gathered}
$$

where the state vector $x_{t}=\left(x_{1}, x_{2}, \ldots, x_{n}\right)^{T}$, the component-wise stochastic differential equation is

$$
d x_{i}(t)=f_{i}\left(x_{t}, t\right) d t+\sum_{\phi} G_{i \phi}\left(x_{t}, t\right) d B_{\phi},
$$

and

$$
\begin{gathered}
\hat{x}_{i}=E\left(x_{i}(t) \mid x_{t_{0}}, t_{0}\right), P_{i j}=E\left(\tilde{x}_{i} \tilde{x}_{j} \mid x_{t_{0}}, t_{0}\right), \\
\tilde{x}_{i}=x_{i}-E\left(x_{i} \mid x_{t_{0}}, t_{0}\right), \overbrace{f_{i}\left(x_{t}, t\right)}^{\Lambda}=E\left(f_{i}\left(x_{t}, t\right) \mid x_{t_{0}}, t_{0}\right),
\end{gathered}
$$

and $f\left(x_{t}, t\right)$ is the system non-linearity and $G\left(x_{t}, t\right)$ is the dispersion matrix. The dispersion matrix is also known as the process noise coefficient matrix in mathematical control theory. The mean and variance evolutions using the third-order approximation can be derived involving the following: (i) first, develop the conditional moment evolution $d \hat{\phi}\left(x_{t}\right)$ using the Fokker-Planck equation (ii) secondly, the mean and variance evolutions can be derived by considering $\phi\left(x_{t}\right)$ as $x_{i}$ and $\tilde{x}_{i} \tilde{x}_{j}$ respectively (iii) finally, the third-order partials of the system non-linearity and diffusion coefficient are introduced into the exact evolution equations (31) and (32). Thus, we have

$$
\begin{gathered}
d \hat{x}_{i}=\left(f_{i}\left(\hat{x}_{t}, t\right)+\frac{1}{2} \sum_{p, q} P_{p q} \frac{\partial^{2} f_{i}\left(\hat{x}_{t}, t\right)}{\partial \hat{x}_{p} \partial \hat{x}_{q}}\right) d t \\
\left(d P_{t}\right)_{i j}=\left(\sum_{p} P_{i p} \frac{\partial f_{j}\left(\hat{x}_{t}, t\right)}{\partial \hat{x}_{p}}+\sum_{p} P_{j p} \frac{\partial f_{i}\left(\hat{x}_{t}, t\right)}{\partial \hat{x}_{p}}+\frac{1}{2} \sum_{p, q, r} P_{i p} P_{q r} \frac{\partial^{3} f_{j}\left(\hat{x}_{t}, t\right)}{\partial \hat{x}_{p} \partial \hat{x}_{q} \partial \hat{x}_{r}}\right. \\
+\frac{1}{2} \sum_{\mathrm{p}, \mathrm{q}, \mathrm{r}} \mathrm{P}_{\mathrm{jp}} \mathrm{P}_{\mathrm{qr}} \frac{\partial^{3} \mathrm{f}_{\mathrm{i}}\left(\hat{\mathrm{x}}_{\mathrm{t}}, \mathrm{t}\right)}{\partial \hat{\mathrm{x}}_{\mathrm{p}} \partial \hat{\mathrm{x}}_{\mathrm{q}} \partial \hat{\mathrm{x}}_{\mathrm{r}}}+\left(\mathrm{GG}^{\mathrm{T}}\right)_{\mathrm{ij}}\left(\hat{\mathrm{x}}_{\mathrm{t}}, \mathrm{t}\right)+\frac{1}{2} \sum_{\mathrm{p}, \mathrm{q}} \mathrm{P}_{\mathrm{pq}} \frac{\partial^{2}\left(\mathrm{GG}^{\mathrm{T}}\right)_{\mathrm{ij}}\left(\hat{\mathrm{x}}_{\mathrm{t}}, \mathrm{t}\right)}{\left.\partial \hat{\mathrm{x}}_{\mathrm{p}} \partial \hat{\mathrm{x}}_{\mathrm{q}}\right) \mathrm{dt} .}
\end{gathered}
$$

The moment evolution equations using the second-order approximation can be found in Jazwinski (1970, p. 363) and become a special case of the evolution equations, i.e. (33) and 
(34). The evolutions of the $i$-th component of the mean vector and $(i, j)$ element of the variance matrix, resulting from combining equations (28), (33), (34), are

$$
\begin{aligned}
& d \hat{\xi}_{i}=A_{i}\left(\hat{\xi}_{t}, P_{t}, t\right) d t, \\
& d P_{i j}=B_{i j}\left(\hat{\xi}_{t}, P_{t}, t\right) d t,
\end{aligned}
$$

where $A=\left(A_{i}\right), B=\left(B_{i j}\right)$ with $1 \leq i \leq 2,1 \leq j \leq 2$ and

$$
\begin{gathered}
A_{1}=\widehat{x}_{2} \\
A_{2}=\alpha \widehat{x}_{1}+\beta \widehat{x}_{2}-a \widehat{x}_{1}^{3}-b \widehat{x}_{2} \widehat{x}_{1}^{2}+\left(-3 a \widehat{x}_{1}-b \widehat{x}_{2}\right) P_{11}+\left(-2 b \widehat{x}_{1}\right) P_{12}, \\
B_{11}=2 P_{12}, \\
B_{12}=B_{21}=P_{11}\left(\alpha-3 a \widehat{x}_{1}^{2}-2 b \widehat{x}_{1} \widehat{x}_{2}\right)+P_{12}\left(\beta-b \widehat{x}_{1}^{2}\right)+P_{22}-3 a P_{11}^{2}-3 b P_{11} P_{12}, \\
B_{22}=2 P_{12}\left(\alpha-3 a \widehat{x}_{1}^{2}-2 b \widehat{x}_{1} \widehat{x}_{2}\right)+2\left(\beta-b \widehat{x}_{1}^{2}\right) P_{22}-6 a P_{11} P_{12}-4 b P_{12}^{2}-2 b P_{11} P_{22} \\
+\sigma_{B}{ }^{2} \widehat{x}_{1}^{2 n}+\frac{1}{2}(2 n)(2 n-1) \sigma_{B}^{2} P_{11} \widehat{x}_{1}^{2 n-2} .
\end{gathered}
$$

Evolution equations (33) and (34) involve the partial differential equation formalism. The mean and variance evolutions for the stochastic problem of concern here become the special cases of equations (33) and (34) as well as assume the structure of ODEs.

\section{Numerical simulations}

Approximate evolution equations, equations (33) and (34), are intractable theoretically, since the global properties are replaced with the local. Numerical experiments under a variety of conditions allow examining the effectiveness of the approximate estimation procedure. The following set of initial conditions and system parameters can be chosen for the numerical testing:

$$
\begin{aligned}
& \alpha=-1, a=0.001, \beta=-0.2, b=0.8, \sigma_{B}=0.028, \sigma_{u}=0.07, \hat{x}_{1}(0)=0.1, \hat{x}_{2}(0)=0.5, \\
& P_{11}(0)=1, P_{12}(0)=0, P_{22}(0)=2, n=3 .
\end{aligned}
$$

Here the initial variances are chosen 'non-zero' and covariances take zero values, which illustrate uncertainties in initial conditions and the uncertainties are initially uncorrelated respectively. The order $n$ of the state-dependent perturbation $\sigma_{B} x_{t}^{n} d B_{t}$ is three, since this choice of the order contributes to higher-order partials of the diffusion coefficient $\left(G G^{T}\right)\left(x_{t}, t\right)$ and allows to examine the efficacy of higher-order estimation algorithms. 
Other choices can be made about the 'state-dependent perturbation order' provided $n \geq 1$. The diffusion parameters $\sigma_{B}$ and $\sigma_{u}$ are selected so that the contribution to the force from the random forcing term is smaller than the contribution from the deterministic part. Thanks to a pioneering paper of $\mathrm{H}$. J. Kushner on stochastic estimation theory that the initial data can be adjusted for the convenience of the estimation procedure, however, it must be tested under a variety of conditions (Kushner 1967, p. 552). The choice of an estimation procedure is also dictated by some experimentation and guesswork. More over, the scanty numerical evidence will not suffice to adjudge the usefulness of the estimation procedure. As a result of these, numerical experiments of this chapter encompass three different approximations.

In this chapter, the three different estimation procedures are the third-order, second-order, and first-order approximate evolution equations. The third-order approximate variance evolution equation involves the additional correction terms, i.e.

$$
\frac{1}{2} \sum_{p, q, r} P_{i p} P_{q r} \frac{\partial^{3} f_{j}\left(\hat{\xi}_{t}, t\right)}{\partial \hat{\xi}_{p} \partial \hat{\xi}_{q} \partial \hat{\xi}_{r}} \text { and } \frac{1}{2} \sum_{p, q, r} P_{j p} P_{q r} \frac{\partial^{3} f_{i}\left(\hat{\xi}_{t}, t\right)}{\partial \hat{\xi}_{p} \partial \hat{\xi}_{q} \partial \hat{\xi}_{r}} \text {. }
$$

In the second-order variance evolution equation, these additional terms are not accounted for. The structure of the second-order mean evolution will be the same as the third-order, since the third-order moment vanishes with 'nearly Gaussian assumption'. The graphs of this chapter illustrate unperturbed trajectories correspond to the bilinear approximation, since the mean trajectories involving the bilinear approximation do not involve the variance term. On the other hand, the perturbed trajectories correspond to the second-order and third-order approximations, see figures (1)-(2). The qualitative analysis of the stochastic problem of concern here confirms the 'mean evolution pattern' using the third-order approximation. This chapter discusses briefly about the numerical simulation of the stochastic Duffing-van der Pol system. A greater detail about the Fokker-Planck analysis of the stochastic problem considered here can be found in a paper recently published by one of the Authors (Sharma 2008).

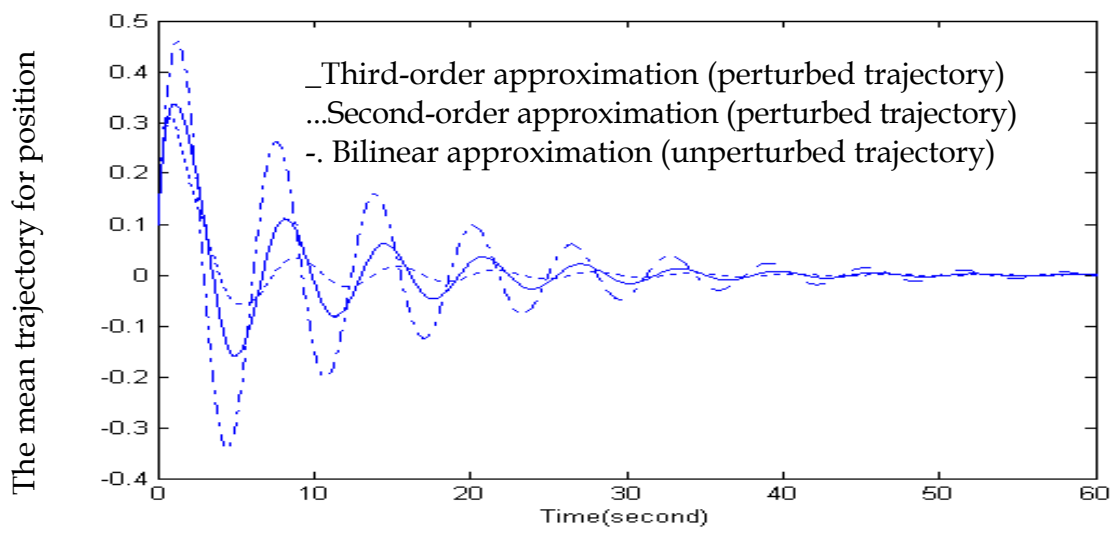

Fig. 1. A comparison between the mean trajectories for position using three approximations 


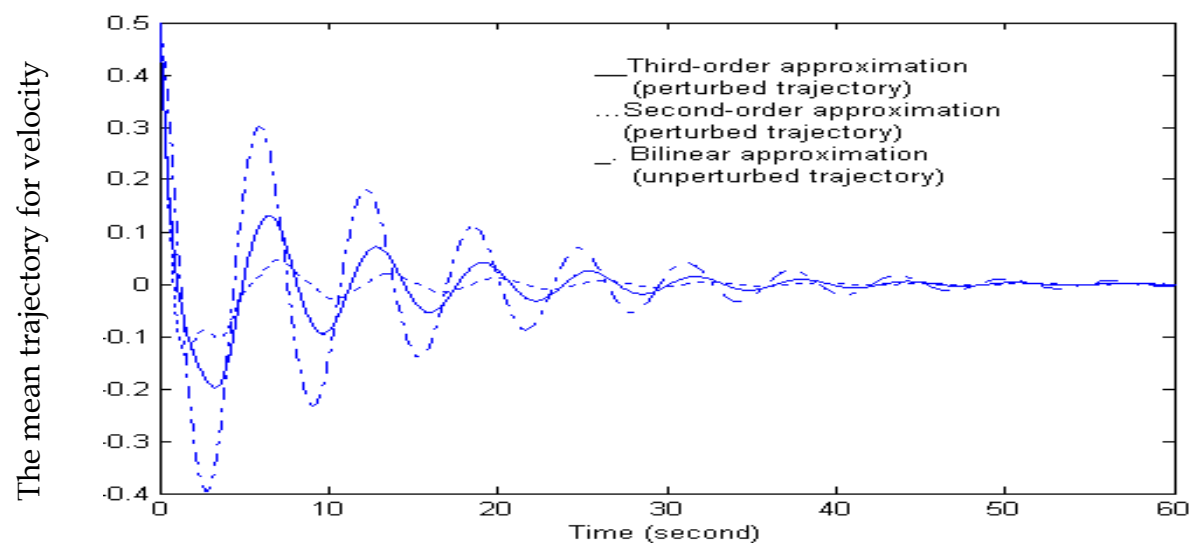

Fig. 2. A comparison between the mean trajectories for velocity using three approximations

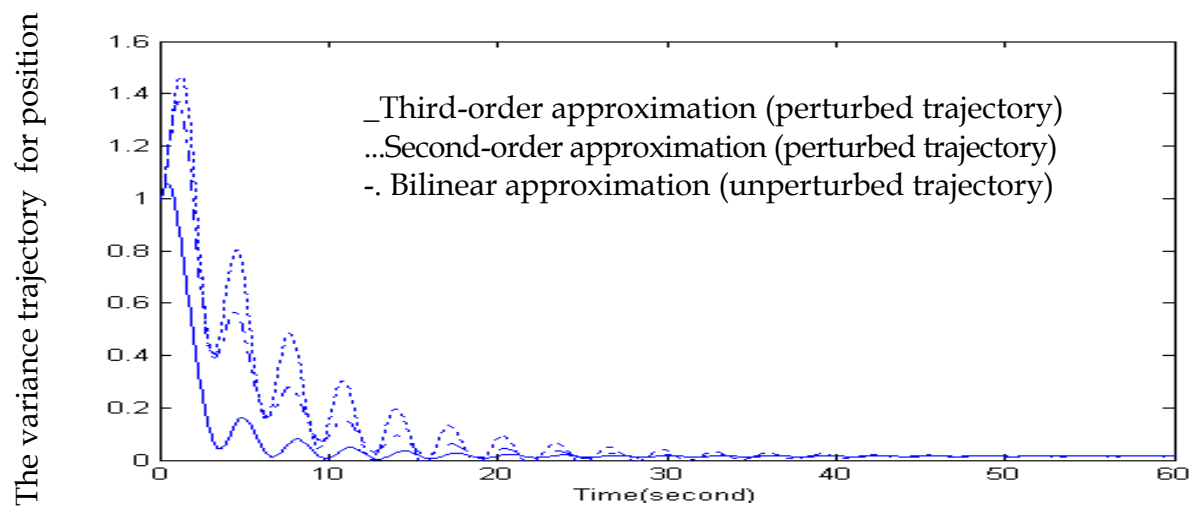

Fig. 3. A comparison between the variance trajectories for position using three approximations

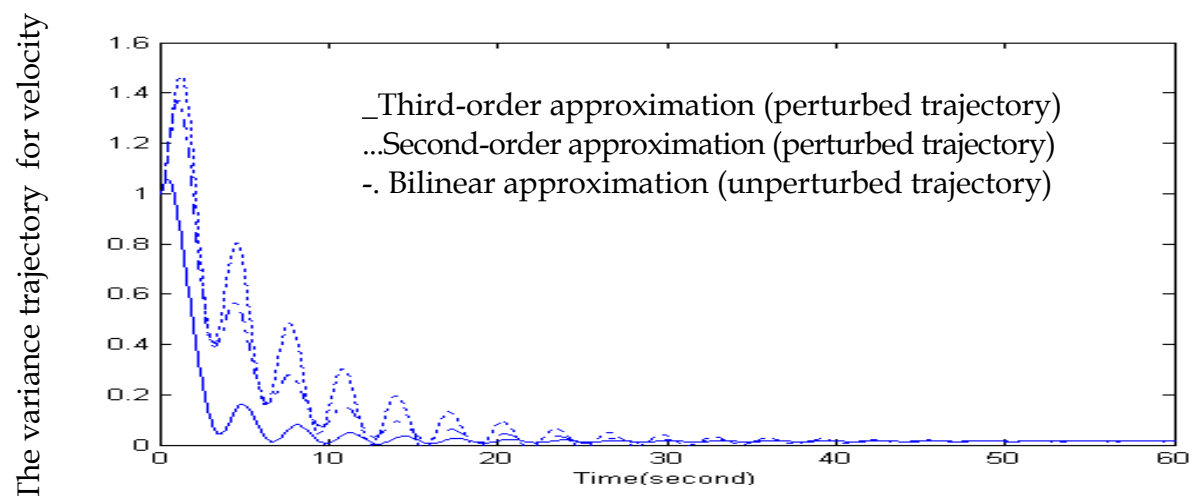

Fig. 4. A comparison between the variance trajectories for velocity using three approximations 


\section{Conclusion}

In this chapter, the Authors have summarized four different methods to derive the FokkerPlanck equation, including two elementary proofs. The Fokker-Planck equation of the OU process-driven stochastic differential system, which received relatively less attention in literature, is also discussed. Most notably, in this chapter, the OU process with non-zero, finite and smaller correlation time was considered. This chapter discusses briefly approximate methods to the Fokker-Planck equation, stochastic differential equations as well as lists 'celebrated books' on the topic. It is believed that the Fokker-Planck analysis of the stochastic problem discussed here will be useful for analysing stochastic problems from diverse field.

\section{Acknowledgement}

One of the Authors of this chapter was exposed to this topic by Professor Harish Parthasarathy, a signal processing and mathematical control theorist. The Authors express their gratefulness to him.

\section{References}

Feller, W. (2000). An Introduction to Probability Theory and its Applications, John Wiley and Sons, New York and Chichester, vol.2.

Fletcher, C. A. J. (1984). Computational Galerkin Methods, Springer-Verlag, New York, Berlin.

Hänggi, P. (1995). The functional derivative and its use in the description of noisy dynamical systems, Stochastic Processes Applied to Physics (L. Pesquera and M. Rodriguez, eds.), World Scientific, Heyden, Philadelphia, pp. 69-95.

Hwalisz, L., Jung, P., Hänggi, P., Talkner, P. \& Schimansky-Geier, L. (1989). Colored noise driven systems with inertia, Z. Physik B, 77, 471-483.

Jazwinski, A. H. (1970). Stochastic Processes and Filtering Theory, Academic Press, New York and London.

Kloeden, P. E. and Platen, E. (1991). The Numerical Solutions of Stochastic Differential Equations (Applications of Mathematics), Springer, New York.

Kowalski, K. \& Steeb W-H. (1991). Non-Linear Dynamical Systems and Carleman Linearization, World Scientific, Singapore, New Jersy.

Kushner, H. J. (1967). Approximations to optimal non-linear filters, IEEE Trans. Automat. Contr. 12(5), 546-556.

Liptser, R. S. and Shiryayev, A. N. (1977). Statistics of Random Processes 1, Springer, Berlin.

Risken, H. (1984). The Fokker-Planck Equation: Methods of Solution and Applications, SpringerVerlag, Berlin.

Sage, A. P. and Melsa, M. L. (1971). Estimation Theory with Applications to Communications and Control, Mc-Graw Hill, New York.

Sharma, Shambhu N. (Oct. 2008). A Kolmogorov-Fokker-Planck approach for a stochastic Duffing-van der Pol system, Differential Equations and Dynamical Systems (An international Journal for theory, applications and computer simulations), 16(4), 351377. DOI: 10.1007/s12591-008-0019-x.

http://www.springerlink.com/content/t5315t2k62154151/ 


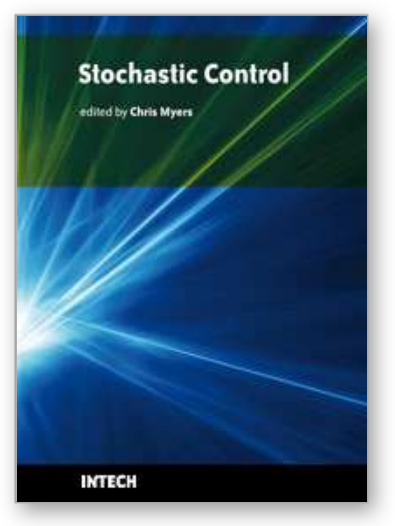

\author{
Stochastic Control \\ Edited by Chris Myers
}

ISBN 978-953-307-121-3

Hard cover, 650 pages

Publisher Sciyo

Published online 17, August, 2010

Published in print edition August, 2010

Uncertainty presents significant challenges in the reasoning about and controlling of complex dynamical systems. To address this challenge, numerous researchers are developing improved methods for stochastic analysis. This book presents a diverse collection of some of the latest research in this important area. In particular, this book gives an overview of some of the theoretical methods and tools for stochastic analysis, and it presents the applications of these methods to problems in systems theory, science, and economics.

\title{
How to reference
}

In order to correctly reference this scholarly work, feel free to copy and paste the following:

Shambhu N. Sharma and Hiren G. Patel (2010). The Fokker-Planck Equation, Stochastic Control, Chris Myers (Ed.), ISBN: 978-953-307-121-3, InTech, Available from: http://www.intechopen.com/books/stochasticcontrol/the-fokker-planck-equation

\section{INTECH}

open science | open minds

\author{
InTech Europe \\ University Campus STeP Ri \\ Slavka Krautzeka 83/A \\ 51000 Rijeka, Croatia \\ Phone: +385 (51) 770447 \\ Fax: +385 (51) 686166 \\ www.intechopen.com
}

\author{
InTech China \\ Unit 405, Office Block, Hotel Equatorial Shanghai \\ No.65, Yan An Road (West), Shanghai, 200040, China \\ 中国上海市延安西路65号上海国际贵都大饭店办公楼 405 单元 \\ Phone: +86-21-62489820 \\ Fax: +86-21-62489821
}


(C) 2010 The Author(s). Licensee IntechOpen. This chapter is distributed under the terms of the Creative Commons Attribution-NonCommercialShareAlike-3.0 License, which permits use, distribution and reproduction for non-commercial purposes, provided the original is properly cited and derivative works building on this content are distributed under the same license. 